\title{
EXPRESSION PROFILING OF SCN8A AND NDUFC2 GENES IN COLORECTAL CARCINOMA
}

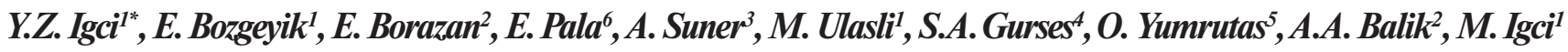 \\ ${ }^{1}$ University of Gaziantep, Faculty of Medicine, Department of Medical Biology, Gaziantep 27310, Turkey \\ ${ }^{2}$ University of Gaziantep, Faculty of Medicine, Department of General Surgery, Gaziantep 27310, Turkey \\ ${ }^{3}$ Adiyaman Education and Research Hospital, Adiyaman 02200, Turkey \\ ${ }^{4}$ Zirve University, Faculty of Medicine, Department of Medical Biology, Gaziantep 27260, Turkey \\ ${ }^{5}$ Adiyaman University, Faculty of Medicine, Department of Medical Biology, Adiyaman 02040, Turkey \\ ${ }^{6}$ Sanko University, Faculty of Medicine, Department of Medical Biology, Gaziantep 27090, Turkey
}

\begin{abstract}
Aim: The expression differences of $S C N 8 A$ (which encodes type VIII alpha subunit of voltage gated sodium channel) and NDUFC2 (which encodes $\mathbf{C} 2$ subunit of Complex I enzyme in oxidative phosphorylation) genes were evaluated in paired colorectal cancer (CRC) tissues which was relied on our partial transcriptome analysis data in cancer cell lines. Materials and Methods: A total of 62 paired tissues of CRC patients (34 male, 28 female) were included in the study. The mRNA levels of $S C N 8 A$ and $N D U F C 2$ genes were determined by using real-time PCR (qRT-PCR and semiquantitative PCR). Results: SCN8A gene expression level was significantly lower in tumor tissues $(p=0.0128)$ and in the patients with the age below 45 years $(p=0.0049)$. There were also meaningful relationships between the gender, grade of CRC, tumor location, histopathological classification, and SCN8A expression. There was no NDUFC2 differential expression. However, the tumors taken from right colon had significantly lower NDUFC2 expression. Conclusion: Although the voltage gated sodium channels (VGSCs) and Complex I (CI) were associated to a number of diseases including different types of cancers, the different subunits of CI and individual members of VGSCs seem to be cancer type-specific in varying proportions.
\end{abstract} Key Words: colorectal carcinoma, $S C N 8 A$, NDUFC2, Complex I, voltage gated sodium channels, gene expression.

Colorectal cancer $(\mathrm{CRC})$ is one of the most common types of cancer worldwide [1]. In the United States, CRC is the third in the leading causes of death due to cancer among men and women [1]. Thirty percent of CRC cases have familial basis while $67 \%$ are sporadic, and the rest $3 \%$ of the cases have inherited CRC syndromes. The complex etiology of sporadic $\mathrm{CRC}$ involves a combined effect of genetic and environmental risk factors [2].

Various genes are affected in terms of expression during the multistep malignant transformation into CRC [3]. Among those genes, plasma membrane ion channel encoding genes are of special importance since they are involved in intracellular signaling events and they activate specific cellular responses. However, ion channels research is considered relatively novel in cancer studies [4] and the transcriptional regulation is not well characterized for the sodium channel genes [5]. The evidences suggesting the correlation of ion channels with cancer are accumulating and the voltage gated sodium channels (VGSCs) were associated to invasion and metastasis behaviors of several types of cancers [4, 6-8]. Among those channels, sodium channel, voltage gated, type VIII, alpha subunit $\left(\mathrm{Na}_{\mathrm{v}} 1.6\right)$ is encoded by $S C N 8 A$ gene. $S C N 8 A$ has specific roles in neurons and skeletal and

Submitted: January 20, 2015

*Correspondence: $\quad$ Fax: +90 342 3603928;

E-mail: igci@gantep.edu.tr

Abbreviations used: $\mathrm{Cl}$ - Complex I; $\mathrm{Cp}$ - crossing point; CRC colorectal cancer; GAPDH - glyceraldehyde-3-phosphate dehydrogenase; $\mathrm{Na}_{v} 1.6$ - sodium channel, voltage gated, type VIII, alpha subunit; qRT-PCR - real-time PCR; VGSCs - the voltage gated sodium channels. cardiac muscles [5]. The gene is located on $12 q 13$ and the transcript variants of this gene encode two distinct proteins which are 1980 and 1939 amino acids (aa) in length (http://www.ncbi.nlm.nih.gov/gene/6334). An approximate 40 -fold overexpression of SCN8A gene was detected in cervical cancer samples [9]. Another significant increase in SCN8A mRNA expression in prostate cell lines was detected, as well [10].

Complex I (Cl), NADH: ubiquinone oxidoreductase is of critical importance for cell physiology and is the first multiprotein complex involved in the formation of the proton-motive force in mitochondria. Its main function is to transport the electrons by oxidation of NADH followed by reduction of ubiquinone [11, 12]. The genes encoding various $\mathrm{Cl}$ subunits were associated with diseases including severe neonatal lactic acidosis, Leigh syndrome, neonatal cardiomyopathy with lactic acidosis, leukodystrophy with macrocephaly, and hepatopathy with renal tubulopathy $[12,13]$ and malignancies including breast, colon and thyroid cancers [14-17]. Human $\mathrm{Cl}$ is composed of 45 subunits making it the largest and most complicated enzyme in the oxidative phosphorylation system. Thirty-nine of the subunits of mammalian $\mathrm{Cl}$ are encoded by the nuclear genome and the rest is encoded by the mitochondrial genome [13]. Among those, NDUFC2 gene is located on 11q14.1 and the transcript variants of this gene encode three distinct proteins which are 119, 88, and 96 aa. in length (http://www.ncbi.nlm.nih.gov/gene/4718). The downregulation of NDUFC2 gene in papillary thyroid carcinoma was previously reported [16]. The chromosomal cytoband region 11q14.1 spanning NDUFC2 gene was shown as amplified in estrogen receptor nega- 
tive breast cancer and this region was associated with worse prognosis of this disease, as well [15]. Additionally, NDUFC2 gene was suggested as being one of the candidate genes involved in microsatellite instability of colon adenomas [14].

SCN8A and NDUFC2 genes are members of different and almost unrelated pathways which also are of importance in terms of CRC development and progression. However, this pilot study was relied on our partial transcriptome analysis results in which we determined altered expression of SCN8A and NDUFC2 genes in various cancer cell lines. Current study was planned to confirm our previous findings and it is aimed to evaluate the gene expression differences of SCN8A and NDUFC2 genes in CRC tissues.

\section{MATERIALS AND METHODS}

Subjects and sample collection. A total

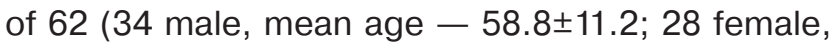
mean age $-53.8 \pm 12.5)$ CRC patients who underwent surgery were included in the study. Paired tumor and tumor-surrounding normal tissues of the patients were used. Samples were placed into liquid nitrogen immediate after the surgery and kept in $-80{ }^{\circ} \mathrm{C}$ until RNA isolation.

Fifty-four of the samples were adenocarcinoma while 8 were mucinous adenocarcinoma, according to the histopathological classification. Six of the tumors were located in cecum, 12 in right colon, 2 in transverse colon, 11 in left colon, 10 in sigmoid colon, and 21 were located in rectum.

This study was approved by the local ethical committee in concordance with the declaration of Helsinki (University of Gaziantep, Clinical research ethical committee, protocol \# 219/11.06.2013). Written consent was taken from all of the patients.

RNA isolation and gene expression analysis. RNA isolation was made by using RNA isolation kit (QIAGEN, Germany) according to the instructions of the manufacturer. RNA concentrations were determined by using spectrophotometer (NanoDrop, ND1000, USA). cDNA synthesis was performed by using AMV first strand cDNA synthesis kit (New England Biolabs, UK) according to the instructions of the manufacturer. Expression primers spanning SCN8A and NDUFC2 mRNAs were designed by using Primer-BLAST (http://www.ncbi.nlm.nih.gov/tools/primerblast/). Primer sequences were presented in Table 1. GAPDH (glyceraldehyde-3-phosphate dehydrogenase) gene was used as the housekeeping gene used for normalization of the expression results of the genes of interest.

Table 1. The characteristics of the primer pairs used for expression analysis

\begin{tabular}{clcc}
\hline \multirow{2}{*}{ Gene } & \multicolumn{1}{c}{ Primer sequence } & $\begin{array}{c}\text { Product } \\
\text { length (bp.) }\end{array}$ & $\begin{array}{c}\text { Annealing tem- } \\
\text { perature }\left({ }^{\circ} \mathrm{C}\right)\end{array}$ \\
\hline \multirow{2}{*}{ NDUFC2 } & $\begin{array}{l}\text { F: TTGCTTCTAATTGCACCAAGCTC } \\
\text { R: AGAAGGTGGTTTCGAAGGAGG }\end{array}$ & 150 & 58 \\
\multirow{2}{*}{ SCN8A } & $\begin{array}{l}\text { F: CCAAACTAAAGGTGCACGCC } \\
\text { R: TGGAAGTCACCATTCCGGTG }\end{array}$ & 148 & 58 \\
GAPDH & $\begin{array}{l}\text { F: GGTCCACCACCCTGTTGCTGT } \\
\text { R: AGACCACAGTCGATGCCATCAC }\end{array}$ & 456 & 65 \\
\hline
\end{tabular}

Gene expression levels were determined by using real-time PCR (qRT-PCR) on LightCycler ${ }^{\circledR} 480$ instrument. PCR conditions were as follows: 2 min at $50{ }^{\circ} \mathrm{C}$, 10 min at $95^{\circ} \mathrm{C}, 40$ cycles of 15 s at $95^{\circ} \mathrm{C}, 30$ s at $60^{\circ} \mathrm{C}$, and $30 \mathrm{~s}$ at $72^{\circ} \mathrm{C}$. Semi-quantitative PCR analysis was conducted prior to qRT-PCR analysis in order to confirm the findings (Fig. 1). Thermal conditions were as follows; $5 \mathrm{~min}$ at $95^{\circ} \mathrm{C}, 35$ cycles of $30 \mathrm{~s}$ at $95^{\circ} \mathrm{C}$, $30 \mathrm{~s}$ at appropriate annealing temperature presented in Table $1,30 \mathrm{~s}$ at $72{ }^{\circ} \mathrm{C}$ and $5 \mathrm{~min}$ at $72{ }^{\circ} \mathrm{C}$.
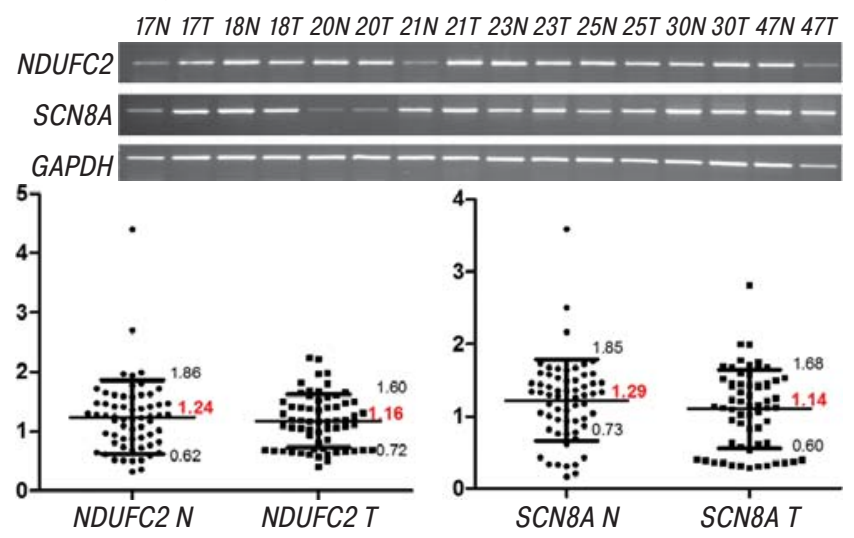

Fig. 1. Semi-quantitative PCR results of NDUFC2 and $S C N 8 A$ genes. Top: Two percent agarose gel image of randomly selected 8 samples' results were shown. GAPDH gene was utilized for normalization of the expression values by using. Normal $\left.\right|_{\text {Target Gene/ }}$ Reference Gene-Tumor ${ }_{\text {Target Gene/Reference Gene }}$ formula. Bottom: The graphs show the average expression levels of the samples. The intensity of the bands were quantitated and densitometric analysis was made by using ImageJ software ( $\mathrm{v} 1.46 \mathrm{r}$ ). A general decrease in the expression values of NDUFC2 and SCN8A genes in tumor tissues compared to normal tissues were prominent.

NDUFC2; Mean, N:1,24, T:1,16; Std. Deviation, N:0,62, T:0,44; Std. Error, N:0,079, T:0,056

SCN8A; Mean, N:1,29, T:1,14; Std. Deviation, N:0,56, T:0,54; Std. Error, N:0,072, T:0,069

Statistical analysis. After normalization by using GAPDH expression, Cp (Crossing point) values were used for the statistical analysis. Calculation was made by using $2^{-\triangle \mathrm{Cp}}$ formula as previously described [18]. The expression levels of SCN8A and NDUFC2 in tumor and paired normal tissues were compared. The relationships between age, gender, grade of the tumor, tumor location, histopathological classification, and gene expression levels were also evaluated. Wilcoxon test was used for the statistical analyses by using GraphPad Prism (v.5) software. All statistical tests were two-tailed and $p<0.05$ was considered as significant.

\section{RESULTS}

SCN8A gene expression level was significantly lower in tumor tissues compared to paired normal tissues ( $p=0.0128$ ) (Table 2, Fig. 2). The decrease in expression was much more significant in the patients with the age below 45 years $(p=0.0049)$. Of the other patient parameters evaluated, there were also meaningful relationships between the gender (female; $p=0.012$ ), grade of CRC (grade $I ; p=0.02$ ), tumor location (cecum; $p=0.031$ and right colon; $p=0.021$ ), histopathological classification (adenocarcinoma; $\mathrm{p}=0.017$ ) and SCN8A expression CRC (Table 2). 
There was no NDUFC2 gene expression difference between the tumors and paired normal tissues in overall (Fig. 3). However, the tumors taken from right colon had significantly lower NDUFC2 expression when compared to paired normal tissues $(p=0.042)$ (Table 2$)$.

Table 2. The patients characteristics, tumor grades, tumor locations, histopathological classifications and the results of the statistical analyses

\begin{tabular}{|c|c|c|c|c|}
\hline & & Sample & $p \mathrm{~V}$ & lues \\
\hline & Parameter & $\begin{array}{c}\text { count, } \\
\text { n (\%) }\end{array}$ & SCN8A & NDUFC2 \\
\hline Age range & $<45(38.8 \pm 4.19)$ & $11(17.7)$ & 0.0049 & 0.57 \\
\hline & $45-60(53.25 \pm 4.45)$ & $28(45.2)$ & 0.22 & 0.97 \\
\hline & $>60(69.04 \pm 5.4)$ & $23(37.1)$ & 0.3 & 0.84 \\
\hline Sex & Female & $28(45.2)$ & 0.012 & 0.79 \\
\hline & Male & $34(54.8)$ & 0.25 & 0.85 \\
\hline Grade & Grade II & $17(27.4)$ & 0.02 & 0.84 \\
\hline & Grade III & $11(17.7)$ & 0.63 & 0.96 \\
\hline & Grade IV & $34(54.8)$ & 0.15 & 0.87 \\
\hline Tumor loca- & Cecum & $6(9.7)$ & 0.031 & 0.56 \\
\hline tion & Colon & $35(56.4)$ & 0.12 & 0.36 \\
\hline & Right colon & $12(19.4)$ & 0.021 & 0.042 \\
\hline & Transverse colon & $2(3.2)$ & NS & NS \\
\hline & Left colon & $11(17.7)$ & 0.14 & 0.7 \\
\hline & Sigmoid colon & $10(16.1)$ & 0.27 & 0.62 \\
\hline & Rectum & $21(33.9)$ & 0.25 & 0.14 \\
\hline Histopatho- & Adenocarcinoma & $54(87.1)$ & 0.017 & 0.71 \\
\hline $\begin{array}{l}\text { logical classi- } \\
\text { fication }\end{array}$ & Mucinous adenocarcinoma & $8(12.9)$ & 0.54 & 0.25 \\
\hline Total & & $62(100)$ & 0.0128 & 0.97 \\
\hline
\end{tabular}

NS - not significant.

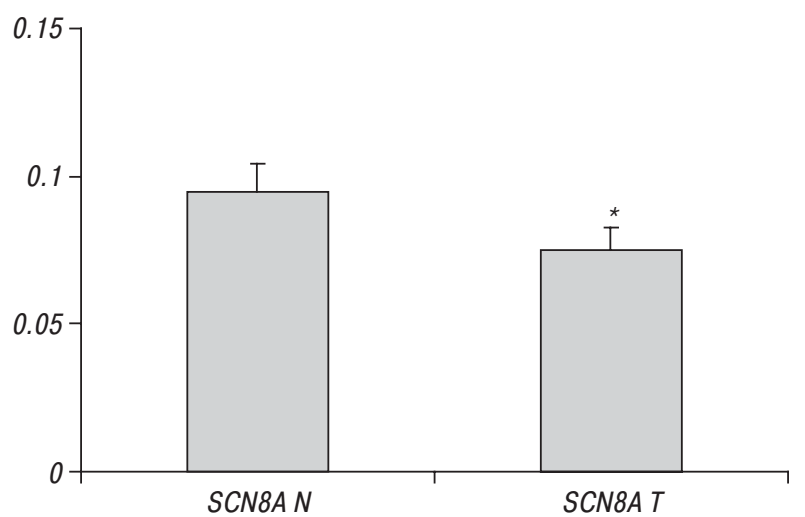

Fig. 2. Calculated $2^{-\triangle C p}$ values of tumor and paired normal tissues for SCN8A gene. $\mathrm{N}$ - normal tissue; $\mathrm{T}$ - tumor tissue; * denotes statistical significance

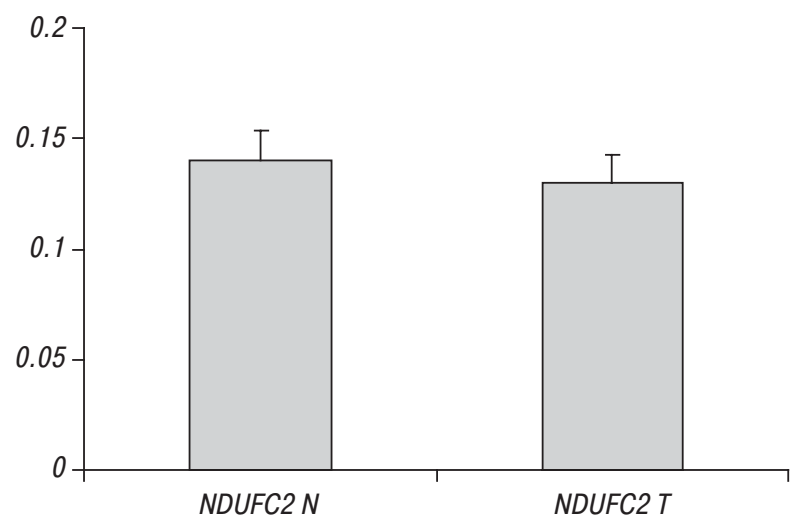

Fig. 3. Calculated $2^{-\Delta \mathrm{Cp}}$ values of tumor and paired normal tissues for NDUFC2 gene. $\mathrm{N}$ - normal tissue; $\mathrm{T}$ - tumor tissue

\section{DISCUSSION}

As of the other types of cancer, the complexity of CRC is a major obstacle in understanding of the genetics of the disease [2]. Therefore, molecular classification of CRC still remains a challenge that researchers have to overcome in the coming years [19].
The implementation of effective $\mathrm{CRC}$ biomarkers may be realized by better understanding of cancer. In our study, we have evaluated the gene expression differences of SCN8A and NDUFC2 genes both of which are involved in different and important cellular processes, in CRC tissues and surrounding healthy colonic mucosa, by using qRT-PCR method. The rationale of the study was based on our preliminary results.

Differential expression and/or genetically alterations of the members of the VGSCs might be involved in the progression of the malignant growth [4]. In our study, the expression of SCN8A gene which encodes $\mathrm{Na}_{v} 1.6$ was significantly lower in CRC samples. Interestingly, this decrease was much more significant in the patients with the age below 45 years. Accumulating data suggest that VGSCs, especially $\mathrm{Na}_{\mathrm{v}} 1.5$, $\mathrm{Na}_{v} 1.6$, and $\mathrm{Na}_{v} 1.7$, were associated with various types of cancers in terms of oncogenic advantage and invasion capacity $[7,10,20,21]$. However, the expression profiles of the individual members of VGSCs are highly variable. In a study aiming to investigate functional expression of alpha subunits of VGSCs in human ovarian cancer; only $\mathrm{Na}_{v} 1.1, \mathrm{Na}_{\mathrm{v}} 1.3, \mathrm{Na}_{\mathrm{v}} 1.4$, and $\mathrm{Na}_{v} 1.5$ mRNAs were overexpressed in ovarian cancers among the 12 members of VGSCs [7]. In the same study, only $\mathrm{Na}_{\mathrm{v}} 1.2, \mathrm{Na}_{\mathrm{v}} 1.4, \mathrm{Na}_{\mathrm{v}} 1.5$, and $\mathrm{Na}_{\mathrm{v}} 1.7$ mRNAs were overexpressed in metastatic ovarian cancers. Taken together, the individual members of VGSCs were differentially expressed specific to cancer type and its malignancy potential. Previous studies reported overexpression of SCN8A gene in cervical cancer and prostate cancers $[9,10]$. However, in our study there was downregulation of SCN8A mRNA in CRC tissues. This decrease, appears to be CRC-specific, may be due to the aforementioned cancer-type dependent expression of VGSCs. The other alpha subunits of VGSCs could also be associated with CRC. Additionally, the alternatively-spliced members of VGSCs may also involve in CRC progression [8]. The overexpression of VGSCs might be an important factor in triggering metastasis and motility due to the altered electrophysiological status of the cell [4]. The altered expressions of VGSCs, either as a whole or as particular members, might be key in developing potential diagnostic or therapeutic targets [10]. Because this was a pilot study, we did not determine the protein expression levels of SCN8A and we did not determine the expression levels of VGSCs other than SCN8A, either. Further studies are needed.

Except for the samples excised from right colon, there was no significant expressional change for NDUFC2 gene in this study. This was contrary to our expectation. This might be due to the heterogenicity of cancer cells. NDUFC2 gene encodes one of the 45 subunits of $\mathrm{Cl}$ [13]. $\mathrm{Cl}$ is a huge complex and it is placed in a complicated physically and functionally interconnected cellular network [12]. In our previous study [16], we reported downregulation of NDUFC2 in papillary thyroid carcinoma. Additionally, $\mathrm{Cl}$ was associated to a number of diseases including different types of cancers $[14,15]$. However, the type 
of $\mathrm{Cl}$ subunit and whether they are encoded from nuclear or mitochondrial genome seems to be disease-specific. Therefore, the evaluation of $\mathrm{Cl}$ subunits as a whole and the protein expressions of these individual subunits are warranted. NDUFC2 protein has three isoforms (http://www.ncbi.nlm.nih.gov/gene/4718). The primer sets used in this study for gene expression analysis of NDUFC2 gene were spanning the shared sequence of all three isoforms. Hence, the expression levels we determined were in overall; rather than isoform-specific. The potential differential expressions of NDUFC2 splice variants should be tested. As the SCN8A gene, NDUFC2 gene expression was decreased for the samples taken out from the right coIon. This data should also be verified in larger cohorts.

$\mathrm{CRC}$ is one of the most common cancers worldwide and diagnosis of CRC could be conducted in a noninvasive manner by the usage of molecular markers. The discovery of new markers and the combinations of them with the others is thought to increase the diagnostic efficiency. This pilot study represents the expression profiling of SCN8A and NDUFC2 genes each of which encode one of the subunits of two important (and unrelated with each other) complexes in terms of $\mathrm{CRC}$ development and progression. Further studies aiming to evaluate the relationship between CRC and other $\mathrm{Cl}$ subunits and other members of VGSCs in terms of mRNA and protein expressions are warranted.

\section{CONFLICT OF INTEREST}

The authors declare that there are no conflicts of interest.

\section{FINANCIAL SUPPORT}

None declared.

\section{REFERENCES}

1. Siegel R, Naishadham D, Jemal A. Cancer statistics, 2013. CA Cancer J Clin 2013; 63: 11-30.

2. Pardini B, Naccarati A, Vodicka P, et al. Gene expression variations: potentialities of master regulator polymorphisms in colorectal cancer risk. Mutagenesis 2012; 27: 161-7.

3. Geyik E, Igci YZ, Pala E, et al. Investigation of the association between ATP2B4 and ATP5B genes with colorectal cancer. Gene 2014; 540: 178-82.

4. Prevarskaya N, Skryma R, Shuba Y. Ion channels and the hallmarks of cancer. Trends Mol Med 2010; 16: 107-21.

5. O'Brien JE, Meisler MH. Sodium channel SCN8A (Nav1.6): properties and de novo mutations in epileptic encephalopathy and intellectual disability. Front Genet 2013; 4: 213.
6. Fraser SP, Pardo LA. Ion channels: functional expression and therapeutic potential in cancer. Colloquium on Ion Channels and Cancer. EMBO Rep 2008; 9: 512-5.

7. Gao R, Shen Y, Cai J, et al. Expression of voltage-gated sodium channel alpha subunit in human ovarian cancer. Oncol Rep 2010; 23: 1293-9.

8. House CD, Vaske CJ, Schwartz AM, et al. Voltage-gated $\mathrm{Na}+$ channel SCN5A is a key regulator of a gene transcriptional network that controls colon cancer invasion. Cancer Res 2010; 70: 6957-67.

9. Hernandez-Plata E, Ortiz CS, Marquina-Castillo B, et al. Overexpression of $\mathrm{NaV} 1.6$ channels is associated with the invasion capacity of human cervical cancer. Int J Cancer 2012; 130: 2013-23.

10. Shan B, Dong M, Tang H, et al. Voltage-gated sodium channels were differentially expressed in human normal prostate, benign prostatic hyperplasia and prostate cancer cells. Oncol Lett 2014; 8: 345-50.

11. Loeffen J, van den Heuvel L, Smeets R, et al. cDNA sequence and chromosomal localization of the remaining three human nuclear encoded iron sulphur protein (IP) subunits of complex I: the human IP fraction is completed. Biochem Biophys Res Commun 1998; 247: 751-8.

12. Chomova M, Racay P. Mitochondrial complex $\mathrm{I}$ in the network of known and unknown facts. Gen Physiol Biophys 2010; 29: 3-11.

13. Janssen RJ, Nijtmans LG, van den Heuvel LP, et al. Mitochondrial complex I: structure, function and pathology. J Inherit Metab Dis 2006; 29: 499-515.

14. Woerner SM, Kloor M, Mueller A, et al. Microsatellite instability of selective target genes in HNPCC-associated colon adenomas. Oncogene 2005; 24: 2525-35.

15. Chin SF, Teschendorff AE, Marioni JC, et al. High-resolution aCGH and expression profiling identifies a novel genomic subtype of ER negative breast cancer. Genome Biol 2007; 8: R215.

16. Igci YZ, Arslan A, Akarsu E, et al. Differential expression of a set of genes in follicular and classic variants of papillary thyroid carcinoma. Endocr Pathol 2011; 22: 86-96.

17. Santidrian AF, Matsuno-Yagi A, Ritland M, et al. Mitochondrial complex I activity and NAD+/NADH balance regulate breast cancer progression. J Clin Invest 2013; 123: 1068-81.

18. Livak KJ, Schmittgen TD. Analysis of relative gene expression data using real-time quantitative PCR and the 2(-Delta Delta C(T)) Method. Methods 2001; 25: 402-8.

19. Sideris M, Papagrigoriadis S. Molecular biomarkers and classification models in the evaluation of the prognosis of colorectal cancer. Anticancer Res 2014; 34: 2061-8.

20. Diss JK, Stewart D, Pani F, et al. A potential novel marker for human prostate cancer: voltage-gated sodium channel expression in vivo. Prostate Cancer Prostatic Dis 2005; 8: 266-73.

21. Brackenbury WJ, Chioni AM, Diss JK, et al. The neonatal splice variant of Nav1.5 potentiates in vitro invasive behaviour of MDA-MB-231 human breast cancer cells. Breast Cancer Res Treat 2007; 101: 149-60. 